\title{
Variability in the fermentation index, polyphenols and amino acids of seeds of rambutan (Nephelium lappaceum $L$.) during fermentation
}

\begin{abstract}
The variability in the fermentation index, polyphenols, and amino acids of rambutan seeds was investigated during fermentation. Results revealed that fermentation index (FI) value $\geq 1$ was achieved on the 4th day of fermentation. While fermentation significantly reduced the levels of total polyphenols (59\%), tannin (60\%), and saponins (33\%), it seems to have a moderate effect on geraniin, corilagin, and a much stronger effect on ellagic, and gallic acids. During fermentation, variability in gallic acid, geraniin, corilagin, and ellagic acid did not show a consistent trend. In contrast, amino acids significantly decreased up to the second day of fermentation. Importantly, amino acids (phenylalanine, tyrosine and leucine) with characteristic bitter taste showed reductions of $20 \%, 30 \%$, and $40 \%$, respectively after 10 days of fermentation. Sucrose, which was the only sugar present in significant concentrations in unfermented seed, was significantly reduced by fermentation.
\end{abstract}

Keyword: Nephelium lappaceum; Fermentation index; Polyphenols; Amino acids; Food analysis; Food composition; Food processing; Nutrient retention 